\title{
A Study of Brainstem Auditory Evoked Responses in Normal Hearing Patients with Tinnitus
}

\author{
G. $\operatorname{Ravikumar}^{1}$ (1) $\cdot$ V. Ashok Murthy ${ }^{1}$
}

Received: 25 April 2015/Accepted: 21 September 2015/Published online: 26 September 2015

(C) Association of Otolaryngologists of India 2015

\begin{abstract}
Tinnitus is thought to be an auditory phenomenon resulting from spontaneous neuronal activity somewhere along the auditory pathway either in the peripheral or central auditory system. The neural abnormalities underlying tinnitus are largely unknown. This study analysis the auditory brainstem responses in normal hearing patients with tinnitus. This study consisted of 100 patients divided into two groups. Group I (Control): 50 Normal hearing patients without tinnitus. Group II (Study): 50 Normal hearing patients complaining of tinnitus. Both groups were submitted to full audiological history taking, otological examination, basic audiologic evaluation and Auditory brainstem responses (ABR) followed by calculation of the absolute latencies of wave I, III and V and interpeak latencies between waves I-III, III-V and I-V. In the study group 20 patients showed abnormal results in at least 1 of the 6 parameters evaluated. The results of absolute latencies of wave I, III and V showed significant prolongation, but the interpeak latencies of waves I-III, III-V and I-V were not significantly prolonged when compared with control group. Our study data showed that there are changes in the central pathways in the study group. The significance of these changes must be investigated with further audiological and neurological tests. We also understand that ABR has to be included in the work up of tinnitus patients whose hearing is within normal parameters.
\end{abstract}

G. Ravikumar

drravikumar2k2@gmail.com

1 Department of ENT, Rajiv Gandhi Institute of Medical Sciences, Kadapa 516 001, India
Keywords Tinnitus - Auditory brainstem response · Absolute latency $\cdot$ Interpeak latency

\section{Introduction}

Tinnitus is a common and disturbing symptom. The pathogenesis and site of origin have yet to be clearly established. It is often a feature of primary ear disease usually associated with hearing loss, but it may also occur in patients with normal hearing $[1,2]$. Investigators in recent research believe that tinnitus is closely related to functional alterations of the central auditory and non-auditory systems in terms of sensation processing [3-5]. While the pathology in the auditory end organs may act as an initiator inducing a series of changes for tinnitus, it is the sustained plastic changes and aberrant activity residing in the sub cortical and cortical structures of the auditory and non-auditory nervous systems that cause the sensation and problem of tinnitus [6,7].

Attempts have been made to understand tinnitus and to investigate its background by means of auditory evoked potentials (AEPs). Those potentials are used to examine the synchronous discharge of fibers in the auditory pathway and identify the presence of abnormal neuronal activity [8]. Auditory brainstem response audiometry can be used in the evaluation of tinnitus patients for a number of reasons, including its objectivity in evaluating the cochlea and the brainstem auditory pathways. ABR also helps in the differentiation of peripheral versus central tinnitus [9].

In the present study we attempted to determine any significant differences exist in ABR parameters in normal hearing patients with tinnitus when compared with normal hearing subjects without tinnitus. 
The purpose of using Auditory brainstem response audiometry in tinnitus patients was an attempt to supplement and thereby extend our knowledge of the nature and origins of tinnitus in normal hearing subjects.

Aim To know the auditory brainstem responses in normal hearing patients with tinnitus.

Study Design Case-control study.

\section{Method}

Inclusion criteria

1. Patients aged between 18 and 45 years.

2. Patients with normal hearing.

3. Patients with tinnitus.

Exclusion criteria

1. Patients with hearing loss.

2. Patients with middle ear disease.

3. Patients with neurological disease.

4. Patients with acoustic trauma.

5. Patients with chronic medical illness.

Study group consists of 50 normal hearing patients with tinnitus and control group consists of 50 normal hearing subjects without tinnitus. After a detailed ENT examination, patients were subjected to conventional audiometric test (Elkon-Multi audiometer and TDH 49 earphones; Elkon private Limited, Mumbai, India) and Impedance audiometry (GSI 38-Auto Tymp). Then these patients were subjected to Brainstem evoked response audiometry. The ABR recordings were obtained at the initial $10 \mathrm{~ms}$ using IHS-single channel system (intelligent hearing system, Miami, Florida, USA) equipment with the patient lying supine position in a well sound treated room.

The parameters studied were the absolute latency of wave I, III and V and the interpeak latencies between waves I-III, III-V and I-V.

For the statistical comparisons, we considered numbers of ears instead of number of patients because some patients complained about a unilateral symptom and the ears were tested individually.

This work included two groups of patients.

Group I 100 Ears of patients without tinnitus-Control group (50 patients forms 100 Ears).

Group II 70 Ears of patients with tinnitus-Study group (40 Ears from $20 \mathrm{~B} / \mathrm{L}$ tinnitus cases, 30 Ears from $30 \mathrm{U} / \mathrm{L}$ tinnitus cases).

The absolute latencies of wave I, III, V and the interpeak latencies (IPLs) between wave I-III, III-V and I-V of study group compared with the absolute latencies of wave I, III, V and the interpeak latencies of wave I-III, III-V and $\mathrm{I}-\mathrm{V}$ of control group. They were considered prolonged if they increased by more than 2 standard deviation from absolute and IPLs in control.

The results of absolute and interpeak latencies compared between study group and Controls by using "Unpaired $T$ test" in SPSS 19.0 statistical software and $P$ values were considered statistically significant when $P<0.05$.

\section{Observations}

Table 1 shows the results of ABR parameters evaluated in the control group. All individual values in all control patients were within the normal limits.

Table 2 shows the results of ABR parameters evaluated in the study group.

Of the 50 patients in the study group $20(40 \%)$ showed abnormalities in at least 1 of the 6 parameters evaluated compared with the normal values in the control group.

\section{Discussion}

Tinnitus is a persistent and often devastating symptom of auditory system. It is the perception of sound without the presence of any external acoustic source. This sound described by the patients as ringing, hissing or buzzing, roaring, rotation of a ceiling fan, radio which is not tuned properly, etc.

Tinnitus is a symptom of primary ear disease and almost associated with hearing loss, but some normal hearing patients also suffering from tinnitus. In our study we were concentrated on the patients with normal hearing with tinnitus. Since there is a common agreement that tinnitus can be also due to an impaired brain process, and there is lack of scientific evidence to prove that tinnitus arises from cochlear damage in normal-hearing patients has

Table 1 Descriptive statistics of auditory brainstem response parameters in the 100 ears of the control group

\begin{tabular}{lllll}
\hline Parameter & Mean $(\mathrm{ms})$ & Standard deviation & \multicolumn{2}{l}{ Range $(\mathrm{ms})$} \\
\cline { 3 - 5 } & \multicolumn{2}{l}{ Minimum } & Maximum \\
\hline \multicolumn{2}{l}{ Absolute latency of waves } \\
I & 1.6625 & 0.21997 & 1.00 & 1.98 \\
III & 3.7490 & 0.16585 & 3.15 & 4.00 \\
V & 5.6424 & 0.19486 & 5.05 & 5.98 \\
Interpeak latency of waves & & \\
I-III & 2.0837 & 0.21511 & 1.62 & 2.80 \\
III-V & 1.8986 & 0.25035 & 1.22 & 2.62 \\
I-V & 3.9832 & 0.29529 & 3.33 & 4.90 \\
\hline
\end{tabular}


Table 2 Descriptive statistics of auditory brainstem response parameters in the 70 ears of the study group

\begin{tabular}{lllll}
\hline Parameter & Mean $(\mathrm{ms})$ & Standard deviation & \multicolumn{2}{l}{ Range $(\mathrm{ms})$} \\
\cline { 3 - 5 } & \multicolumn{2}{l}{ Minimum } & Maximum \\
\hline \multicolumn{2}{l}{ Absolute latency of waves } \\
I & 1.7328 & 0.14489 & 1.00 & 2.01 \\
III & 3.7981 & 0.12203 & 3.40 & 4.15 \\
V & 5.7177 & 0.17568 & 5.08 & 6.17 \\
Interpeak latency of waves & & \\
I-III & 2.0672 & 0.16500 & 1.42 & 2.58 \\
III-V & 1.9211 & 0.20590 & 1.25 & 2.77 \\
I-V & 4.0011 & 0.20656 & 3.45 & 4.85 \\
\hline
\end{tabular}

encouraged us to investigate whether patients with tinnitus show changes in the central auditory pathways.

As suggested by Jastreboff [10], that tinnitus is due to the aberrant activity within the auditory system, and it is interpreted as sound. He states that the permanent activation of the auditory system changes its state and also its properties of central transmission. External signal transmission would thus be modified. To understand the origin of tinnitus and to investigate its background he performed electrophysiological methods. One such method is Auditory brainstem response audiometry.

Ottaviani et al. [11] hypothesized that tinnitus could be related to an altered central auditory processing mechanism. They hypothesized that peripheral and/or central tinnitus could be due to a functional alteration of nerve fibers, and auditory evoked potentials could be used in detecting them. In particular ABR should be able to identify both peripheral and central lesions, naturally linked to the brainstem.

According to the literature, ABR is a useful tool in investigating the anatomical and functional characteristics of the auditory pathway from the end organ to the inferior colliculus and in detecting lesions even if auditory threshold is unaffected.

So in our study to know the site of lesion in the auditory pathway we used ABR as an objective electrophysiological method for assessing the auditory pathways from the auditory nerve to the Brainstem.

Generally there was no significant difference between normal hearing tinnitus patients and normal hearing subjects. This agreed with Barnea et al. [12] and McKee and Stephens [13].

However, ABR absolute and interpeak latencies were prolonged in some tinnitus patients and this agreed with Kehrle et al. [1], Rosenhall and Axelsson [14], Ikner and Hassen [15], Maurizi et al. [11] and Lemaire and Beutter [16].

In our study, different patterns of ABR abnormalities were found in normal hearing tinnitus patients suggesting central auditory pathway affection. The first pattern was the statistically significant prolongation of wave I, III and V absolute latencies which occurred in 18, 17 and $23 \%$ of ears respectively and the $\mathrm{P}$ values were $0.020,0.027$ and 0.011 respectively. Other patterns of abnormalities include prolongation of interpeak latencies between waves I-III in $6 \%$, III-V in $10 \%$ and I-V in $10 \%$ of ears and the P values were $0.592,0.522$ and 0.642 respectively. Despite of these abnormalities in the interpeak latencies, there was no significant prolongation when compared with control group.

In patients with tinnitus, a prolongation of wave I that also affects the late ABR waves is seen in ears with normal hearing. The method used in this study to evaluate cochlear status (conventional audiogram) might not have been sufficiently sensitive for assessing all aspects of cochlear functioning. This lies in the fact that many commonly

Table 3 Comparision of wave latencies between study and control group

\begin{tabular}{|c|c|c|c|c|c|}
\hline \multirow[t]{2}{*}{ Wave } & \multicolumn{2}{|c|}{ Study group latency $(n=70)$} & \multicolumn{2}{|c|}{ Control group latency $(n=100)$} & \multirow[t]{2}{*}{$P$ value } \\
\hline & Mean & SD & Mean & SD & \\
\hline I & 1.7328 & 0.14489 & 1.6625 & 0.21997 & $0.020(\mathrm{~S})$ \\
\hline III & 3.7981 & 0.12203 & 3.7490 & 0.16585 & $0.027(\mathrm{~S})$ \\
\hline V & 5.7177 & 0.17568 & 5.6424 & 0.19486 & $0.011(\mathrm{~S})$ \\
\hline I-III & 2.0672 & 0.16500 & 2.0837 & 0.21511 & $0.592(\mathrm{NS})$ \\
\hline III-V & 1.9211 & 0.20590 & 1.8986 & 0.25035 & $0.522(\mathrm{NS})$ \\
\hline $\mathrm{I}-\mathrm{V}$ & 4.0011 & 0.20656 & 3.9832 & 0.29529 & 0.642 (NS) \\
\hline
\end{tabular}

Table 3 showing absolute latencies of wave I, III and V of cases showed statistically significant prolongation when compared with control group and the $P$ values for absolute latencies of wave I, III and V were $0.020,0.027$ and 0.011 respectively

Despite of abnormalities present in the study group, ABR interpeak latencies in the study group were not significantly prolonged from the control group and the $P$ values for interpeak latencies between waves I-III, III-V and I-V were $0.592,0.522$ and 0.642 respectively

$S$ significant. So statistically there is a significant difference between the cases and controls; NS not significant. There is no statistical significant difference between the groups 
encountered hearing losses initially affect the high frequencies that were not evaluated in this study.

The absolute latency of wave I and III prolongation indicates aberrant neural activity in the cochlear nerve and cochlear nucleus complex of the auditory pathway respectively. The absolute latency of wave $\mathrm{V}$ prolongation indicates some neural abnormality in the lateral lemniscus and inferior colliculus region of the brainstem.

The prolongation of interpeak latencies between waves I-III, III-V and I-V usually reflects an increased neural conduction time in the auditory nerve and the brainstem $[17,18]$. This could be due to permanent activation of auditory system by tinnitus which might change its central transmission which might modify external stimuli transmission.

In our study in the study group out of 50 patients, $40 \%$ of patients showed abnormalities in the ABR parameters. It is reported in literature that $31-40 \%$ of patients with tinnitus and normal hearing or slight hearing loss have abnormalities in the ABR parameters. In rest of the $60 \%$ of patients with tinnitus with normal hearing showed no abnormal prolongation in the ABR parameters studied. Tinnitus is caused by abnormal spontaneous hyperactivity in the auditory pathways [19] and that the absence of abnormal ABR parameters in tinnitus patients might be due to the masking effect of the stimulus that masks the abnormal activity in the central pathways.

\section{Study Limitations}

One limitation of our study was that patients with tinnitus did not undergo any imaging studies.

\section{Conclusions}

In the present study the absolute latency of waves I, III and $\mathrm{V}$ showed statistically significant prolongation in the study group when compared with control group. It signals a lesion in the distal part of the auditory nerve, cochlear nucleus and lateral lemniscus \& inferior colliculus region of the central auditory pathway respectively. It might be due to a functional alteration of nerve fibers in that region of the auditory pathway.

The other abnormalities found in this study were prolonged IPL of waves I-III, III-V, I-V, usually reflects an increased neural conduction time in the auditory nerve and the brainstem. But these abnormalities did not show significant prolongation from control group and also $40 \%$ patients in the study group only showed abnormalities in the absolute and interpeak latencies and rest of the $60 \%$ of patients in the study group showed normal responses.
By this we come to know after analyzing the various parameters that Auditory brainstem response audiometry is most sensitive audiological investigation in interpretation of tinnitus with normal hearing patients.

The Auditory brainstem response results what we obtained from the patients with and without tinnitus in normal hearing individuals are different from one person to another. This suggests impaired neural firing synchronization and transmission in the central auditory pathway in tinnitus patients. These findings also indicate the pathology underlying tinnitus is not the same in every individual, with possible brainstem involvement in some cases.

\section{Key Message}

Auditory brainstem response audiometry is very important for designing the proper management program and selecting the appropriate medication and instrumentation to relieve tinnitus. It also will help to decide the aim of rehabilitation program whether it is designed to restore of homeostasis of brain activity or to act at more peripheral level.

Thus auditory brainstem response audiometry might contribute to the work up of these patients for locating the lesion in auditory pathways and should be done in tinnitus sufferers. Further evaluation of tinnitus patients based on tinnitus severity, duration and character are recommended to provide more understanding of tinnitus problems and to establish appropriate rehabilitation programs.

\section{References}

1. Kehrle HM, Sampaio ALL, Bezerra R, Almeida VF, Oliveira CA (2008) Comparison of auditory brainstem response results in normal-hearing patients with and without tinnitus. Arch Otolaryngol Head Neck Surg 1346:647-651

2. Singh S, Munjal SK, Panda NK (2011) Comparison of auditory electrophysiological responses in normal-hearing patients with and without tinnitus. J Laryngol Otol 125:668-672

3. Weisz N, Wienbruch C, Dohrmann K et al (2005) Neuromagnetic indicators of auditory cortical reorganization of tinnitus. Brain 128:2722-2731

4. Diesch E, Struve M, Rupp A et al (2004) Enhancement of steady state auditory evoked magnetic fields in tinnitus. Eur J Neurosci 19:1093-1104

5. Norena AJ, Eggermont JJ (2003) Changes in spontaneous neural activity immediately after an acoustic trauma: implications for neural correlates of tinnitus. Hear Res 183:137-153

6. Lee CY, Jaw FS, Pan SL, Lin MY, Young YH (2007) Auditory cortical evoked potentials in tinnitus patients with normal audiological presentation. J Formos Med Assoc 106(12):979-985

7. Smits M, Kovacs S, Ridder D, Peeters RP, Hecke P, Sunaert S (2007) Lateralization of functional magnetic resonance imaging 
(FMRI) activation in the auditory pathway of patients with lateralized tinnitus. Neuroradiology 49:669-679

8. Takwa Adly Gabr (2011) Auditory brainstem response audiometry in tinnitus patients. Egypt J Ear Nose Throat Allied Sci $12: 115-120$

9. Shulman A, Seitz MR (1981) Central tinnitus-diagnosis and treatment: observations of simultaneous binaural auditory brain responses with monaural stimulation in the tinnitus patient. Laryngoscope 92(12):2025-2035

10. Jastreboff PJ (1990) Phantom auditory perception (tinnitus): mechanisms of generation and perception. Neurosci Res 8(4):221-254

11. Maurizi M, Ottaviani F, Paludetti G, Almadori G, Tassoni A (1985) Contribution to the differentiation of peripheral versus central tinnitus via auditory brainstem response evaluation. Audiology 24:207-216

12. Barnea G, Attias J, Gold S, Shahar A (1990) Tinnitus with normal hearing sensitivity: extended high frequency audiometry and auditory-nerve brain stem-evoked responses. Audiology 29:36-45

13. McKee GJ, Stephens SDG (1992) An investigation of normally hearing subjects with tinnitus. Audiology 31(6):313-317

14. Rosenhall U, Axelsson A (1995) Auditory brainstem response latencies in patients with tinnitus. Scand Audiol 24(2):97-100

15. Ikner CL, Hassen AH (1990) The effects of tinnitus on ABR latencies. Ear Hear 11(1):16-20

16. Lemaire MC, Beutter $P$ (1995) Brainstem auditory evoked responses in patients with tinnitus. Audiology 34(6):287-300

17. Moller AR (1984) Pathophysiology of tinnitus. Ann Otol Rhinol Laryngol 93:39-44

18. Møller AR, Jannetta PJ (1982) Evoked potentials from the inferior colliculus in man. Electroencephalogr Clin Neurophysiol 53(6):612-620

19. Cahani M, Paul G, Shahar A (1984) Tinnitus asymmetry. Audiology 23(1):127-135 